\title{
Oscillatory Reduction in Option Pricing Formula Using Shifted Poisson and Linear Approximation
}

\author{
Ro'fah Nur Rachmawati ${ }^{1}$, Irene ${ }^{2}$, and Widodo Budiharto ${ }^{1}$ \\ ${ }^{1}$ School of Computer Science, Statistics \& Computer Science Department, Bina Nusantara University, Indonesia \\ ${ }^{2}$ School of Computer Science, Mathematics Department, Bina Nusantara University, Indonesia
}

\begin{abstract}
Option is one of derivative instruments that can help investors improve their expected return and minimize the risks. However, the Black-Scholes formula is generally used in determining the price of the option does not involve skewness factor and it is difficult to apply in computing process because it produces oscillation for the skewness values close to zero. In this paper, we construct option pricing formula that involve skewness by modified Black-Scholes formula using Shifted Poisson model and transformed it into the form of a Linear Approximation in the complete market to reduce the oscillation. The results are Linear Approximation formula can predict the price of an option with very accurate and successfully reduce the oscillations in the calculation processes.
\end{abstract}

\section{Introduction}

Investing in stock market has a lot of risks that could cause losses in the future. One way to minimize the risk or protect the investment is by trading in derivative instruments. If derivatives are used properly, derivatives can help investors to improve their expected return and minimize risk [1]. One example of derivative instruments are widely known and traded is option. Options are contracts that give the right to buy or sell an asset at a specified price within a specified period. For option holder's benefit, calculation of option prices (return/payoff of option trading) is needed. The option price calculation generally uses the Black-Scholes model formula. In [1] states, the Black-Scholes is widely used to calculate the price of the European option because of the analytical solution for European option pricing.

According [2] and [3], Black-Scholes assumes that at the end of period, the price of underlying asset has Lognormal distribution and return can be expressed with $\ln \frac{S_{t}}{S_{t-1}}$ that has normal distribution. According [4], expected return usually has negatively skewness where the curve has a left tail so that most of the data contained on the right. It follows that the Black-Scholes model cannot be used in option pricing with skewness effect and must be adjusted if the return of the underlying assets do not have normal distribution.

Solution of this problem is using the shifted Poisson in complete market condition where the market does not take the risk for options trading [5]. But after this model is applied, oscillatory processes occur that complicate the calculation. So the aim of this paper is to simplify the computing process by construct a Linear Approximation of the Black-Scholes to reduce the oscillation on computing processes.

\section{Assumptions}

Black \& Scholes [6] formulate a formula to calculate the price of call options as follows :

with :

$$
\begin{gathered}
C(S, t)=S N\left(d_{1}\right)-K e^{-r \tau} N\left(d_{2}\right) \\
\text { where } d_{1}=\frac{\ln \left(\frac{S}{K}\right)+\left(r+\frac{\sigma^{2}}{2}\right) \tau}{\sigma \sqrt{\tau}} \text { and } d_{2}=d_{1} \\
-\sigma \sqrt{\tau}
\end{gathered}
$$

$C(S, t)=$ Price of call option depends on $S$ (initial asset prices) dan $t$ (period)

$S=$ Initial asset prices (underlying asset)

$N(d 1)=$ Cumulative value until $d 1$ in normal distribution

$K=$ Exercise price

$\tau=$ Time to maturity date $(T-t)$

$T=$ Maturity date

$N(d 2)=$ Cumulative value in normal distribution

$e^{-r \tau}=$ Continuous rate of interest

$r=$ Rate of interest

$\sigma=$ Volatility of the underlying assets

Black-Scholes formula is used to calculate the European option prices where it is assumed that the stock 
price is a Geometric Brownian motion. Let $S(t)$ is a nondividend stock price in time $t$ and $X(t)$ is a stochastic process with independent and stationary increments and satisfies:

$$
S(t)=S(0) e^{X(t)}, t \geq 0
$$

Mathematically, the European option is defined by a payoff function $\Pi(s) \geq 0$ and maturity date is $\tau$. At time $\tau$, the option holder will receive $\Pi(S(\tau))$. We assume the interest rate $r$ is constant. Then the option price at time $t$ is

$$
e^{-r(\tau-t)} E^{*}[\Pi(S(\tau)) \mid S(t)], 0 \leq t<\tau
$$

* Sign in equation (1) states that the expected value is taken based on equivalent martingale measure appropriated to the observation of stock prices. So the equation price of the underlying asset is

$$
S(t)=e^{-r(\tau-t)} E^{*}[(S(\tau)) \mid S(t)], 0 \leq t<\tau .
$$

Since $X(t)$ is a process that has independent and stationary increments thus, the condition of equation (2) can be stated as

$$
\begin{aligned}
S(0) & =e^{-r(1-0)} E^{*}[S(1) \mid S(0)] S(0) e^{X(0)} \\
& =e^{-r} E^{*}\left[S(0) e^{X(1)} \mid S(0) e^{X(0)}\right] .
\end{aligned}
$$

Note that, $X(0)=0$, we have

$$
\begin{aligned}
S(0) e^{0} & =e^{-r} E^{*}\left[S(0) e^{X(1)} \mid S(0) e^{0}\right] S(0) \\
& =e^{-r} E^{*}\left[S(0) e^{X(1)} \mid S(0)\right] 1 \\
& =\frac{e^{-r} E^{*}\left[S(0) e^{X(1)} \mid S(0)\right]}{S(0)} .
\end{aligned}
$$

We assume, $=S(0) e^{X(1)}, y=S(0)$. Since $x$ and $y$ are the independent events, then

$$
E\left[S(0) e^{X(1)} \mid S(0)\right]=E\left[S(0) e^{X(1)}\right] .
$$

Use the above equation so we have

$$
1=\frac{e^{-r} E^{*}\left[S(0) e^{X(1)}\right]}{S(0)} .
$$

Since $S(0)$ is deterministic

$$
1=e^{-r} E^{*}\left[e^{X(1)}\right]
$$

To simplify notation of the calculation, we use $\tau=1$ and calculate the option price at time $t=0$.

Let $M(z, t)=E\left[e^{z X(t)}\right]$ is moment generating function for $X(t)$ that satisfies:

$$
\begin{aligned}
& M(z, t)=M(z)^{t}, t>0, \text { where } M(z)=M(z, 1) \\
& E[X(t)]=\mu t
\end{aligned}
$$

$$
\begin{gathered}
\operatorname{Var}[X(t)]=\sigma^{2} t \\
E\left[(X(t)-\mu t)^{3}\right]=\gamma t
\end{gathered}
$$

In classical model, $\{X(t)\}$ is Wiener process. And for $t>0$, random variables $X(t)$ has normal distribution with mean $\mu t$, variance $\sigma^{2} t$ and skewness $\gamma=0$. In this paper we discuss if $\gamma \neq 0$. The idea for this skewness values is in the Complete Market martingale measure [7] are equivalent and lead to a unique option price. Unfortunately, the calculation of this option has a problem in $\gamma$ close to zero, because the calculation process produces oscillations that can be seen in Figure 1. For this reason we would replace equality of martingale measure with linear approximation, which contains a calculation of Black-Scholes option price with the adjustment of skewness coefficient $(\gamma)$.

To construct the above idea, let $\{Y(t)\}$ is a stochastic process with independent and stationary increments and satisfies:

$$
\begin{aligned}
E[Y(t)] & =0 \\
\operatorname{Var}[Y(t)] & =t \\
E\left[Y(t)^{3}\right] & =t \\
E\left[e^{z Y(t)}\right] & =e^{t \psi(z)}
\end{aligned}
$$

where, $\psi(z)=\frac{1}{2} z^{2}+\frac{1}{6} z^{3}+\cdots$.

Then we construct three-parameter-family defined as

$$
X(t)=k Y(\lambda t)+\mu t, t \geq 0 .
$$

For $\mu, \sigma^{2}$ and $\gamma$ we use

$$
k=\frac{\gamma}{\sigma^{2}}, \lambda=\frac{\sigma^{6}}{\gamma^{2}}
$$

Since equation (4) and (5) are satisfies, then we have

$$
\begin{aligned}
M(z, t) & =E\left[e^{z X(t)}\right] \\
& =E\left[e^{z(k Y(\lambda t)+\mu t)}\right] \\
& =E\left[e^{z k Y(\lambda t)+\mu t z)}\right] \\
& =\exp [\lambda t \psi(k z)+\mu t z] .
\end{aligned}
$$

Using equation (6), then equation (9) becomes

$$
M(z, t)=\left(\mu z+\frac{1}{2} \sigma^{2} z^{2}+\frac{1}{6} \gamma z^{3}+\cdots\right) .
$$

\section{Discussions}

In modified Black-Sholes formula for determining the option price we use two methods, namely Shifted Poisson models and then transforming into a Linear Approximation form as follows: 


\subsection{Modification of the Black-Scholes Formula Using Shifted Poisson Model}

According [8] and [9] Shifted Poisson Model [10] is used to modify the Black-Scholes formula by letting:

$$
X(t)=k N(t)-c t
$$

As already given in equation (8) then we can calculate the value of $c$,

$$
\begin{aligned}
E[X(t)] & =\mu t(k \lambda-c) t \\
(k \lambda-c) t & =\mu t \\
k \lambda-c & =\mu \\
c & =k \lambda-\mu \\
& =\frac{\gamma}{\sigma^{2}} \cdot \frac{\sigma^{6}}{\gamma^{2}}-\mu \\
& =\frac{\sigma^{4}}{\gamma}-\mu
\end{aligned}
$$

$\{X(t)\}$ equals to the unchanged value of $k$ and $c$, but $\lambda$ replaced with $\lambda^{*}$, which can be selected and satisfy equation (3). Then we get $\lambda^{*}$ as follow

$$
\begin{aligned}
e^{-r} E^{*}\left[e^{X(1)}\right] & =1 \\
e^{-r} E^{*}\left[e^{k N(1)-c .1}\right] & =1 \\
e^{-r} E^{*}\left[e^{k N(1)-c}\right] & =1 \\
e^{-r-c} E^{*}\left[e^{k N(1)}\right] & =1 .
\end{aligned}
$$

We know that $E\left[e^{k N(t)}\right]=\exp \left(\lambda t\left(e^{k}-1\right)\right)$, then we have

$$
\begin{aligned}
& e^{-r-c} \cdot e^{\lambda\left(e^{k}-1\right)}=1 \\
& e^{\left[-r+\lambda^{*}\left(e^{k}-1\right)-c\right]}=1 \\
& \ln \exp \left[-r+\lambda^{*}\left(e^{k}-1\right)-c\right]=\ln 1 \\
& {\left[-r+\lambda^{*}\left(e^{k}-1\right)-c\right]=0} \\
& \lambda^{*}=\frac{r+c}{e^{k}-1} .
\end{aligned}
$$

According to equation (1), then the price at time $t=0$ and $\tau=1$ is

$$
e^{-r(1-0)} E^{*}[\Pi(S(1)) \mid S(0)]
$$

As has been proved in the previous equations, above equation becomes,

$$
e^{-r} E^{*}[\Pi(S(1))]
$$

Because the expected value using the Poisson distribution, the above equations become,

$$
\begin{aligned}
e^{-r} E^{*} & {[\Pi(S(1))]=e^{-r} \sum_{j=0}^{\infty} e^{-\lambda^{*}} \frac{\lambda^{* j}}{j !} \Pi(S(1)) } \\
= & e^{-r} \sum_{j=0}^{\infty} e^{-\lambda^{*}} \frac{\lambda^{* j}}{j !} \Pi\left(S(0) e^{X(1)}\right) \\
& =e^{-r} \sum_{j=0}^{\infty} e^{-\lambda^{*}} \frac{\lambda^{* j}}{j !} \Pi\left(S(0) e^{k j-c}\right) .
\end{aligned}
$$

Calculation of the European call option with exercise price $K$ and the payoff function $\Pi(s)=(s-K)_{+}$, where $x_{+}$indicates a positive part of $x$. Then, $x_{+}=x$ if $x>0$ and $x_{+}=0$ if $x \leq 0$. Then the option price using Shifted Poisson model is

$$
e^{-r} \sum_{j=0}^{\infty} e^{-\lambda^{*}} \frac{\lambda^{* j}}{j !}\left(S(0) e^{k j-c}-K\right)_{+}
$$

with :

$$
\begin{aligned}
& r=\text { Rate of interest } \\
& \lambda=\text { Poisson intensity } \\
& S(0)=\text { Stock Price } \\
& K=\text { Exercise Price } \\
& c=\frac{\sigma^{4}}{\gamma}-\mu .
\end{aligned}
$$

Or to simplify the calculation of equation (12) we can convert as follow

$$
\begin{array}{r}
S(0)\left[1-N\left(\frac{(\kappa+c \tau)}{k} ; \lambda^{*} e^{k} \tau\right)\right] \\
-K e^{r \tau}\left[1-N\left(\frac{(\kappa+c \tau)}{k} ; \lambda^{*} \tau\right)\right]
\end{array}
$$

with :

$$
\begin{aligned}
& S(0)=\text { Stock Price } \\
& K=\text { Exercise Price } \\
& \kappa=\ln \frac{K}{S(0)} \\
& r=\text { Rate of interest } \\
& \lambda^{*}=\frac{r+c}{e^{k}-1} \\
& c=\frac{\sigma^{4}}{\gamma}-\mu \\
& k=\frac{\gamma}{\sigma^{2}} .
\end{aligned}
$$

This results in accordance with [11].

\subsection{Oscillation in Shifted Poisson Model}

But in equation (12) un-continuities occur when $k j-c=0$ for some value of $j$. Using equation (8) and value of $c$, we have

$\gamma_{1,2}=\frac{-\mu \pm \sqrt{\mu^{2}-4\left(\frac{j}{\sigma^{2}}\right)\left(-\sigma^{4}\right)}}{2\left(\frac{j}{\sigma^{2}}\right)}=\frac{-\mu \pm \sqrt{\mu^{2}+4 j \sigma^{2}}}{\frac{2 j}{\sigma^{2}}}$.

The meaning of the above equation is, this equation is a monotone sequence that converges to 0 . And for skewness $(\gamma)$ close to zero, oscillation occur as shown in Figure 1. So the solution to reduce the oscillation is by transforming Shifted Poisson model into Linear Approximation form for option prices that contain the skewness. 


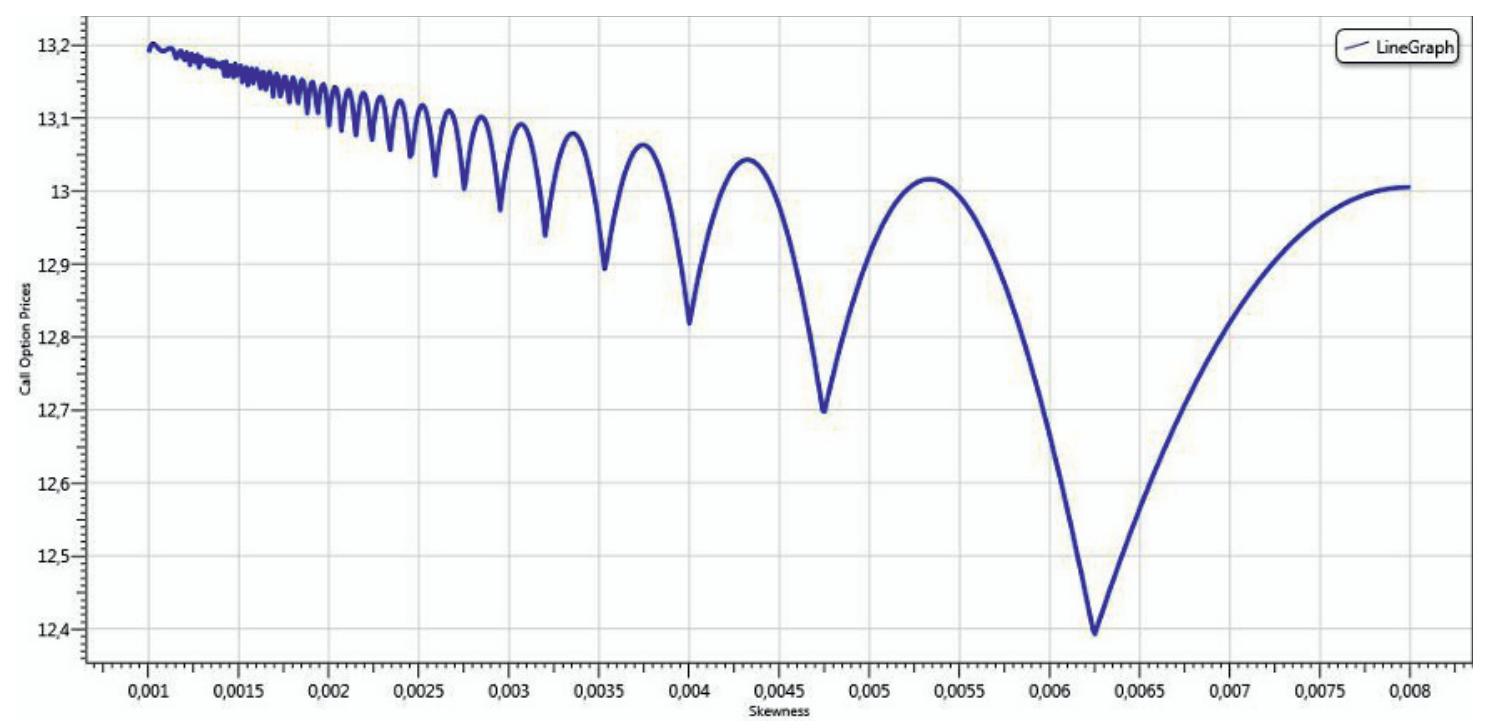

Figure 1. Option Price Calculation Using Shifted Poisson Model.

\subsection{Linear Approximation in Option Price Calculation}

The basic idea of the linear approximation is by using $k$ value containing the skewness in the MacLaurin expansion. Shifted Poisson transformed into the MacLaurin expansion:

$$
\begin{aligned}
\ln E^{*}\left[e^{z X(1)}\right]= & \lambda^{*}\left(e^{k z}-1\right)-c z \\
= & \frac{(r+c)}{e^{k}-1}\left(e^{k z}-1\right)-c z \\
= & r\left(z-\frac{1}{2} k z+\frac{1}{2} k z^{2}+\frac{1}{12} k^{2} z-\frac{1}{4} k^{2} z^{2}+\right. \\
& \left.\frac{1}{6} k^{2} z^{3}+. .\right)+c\left(-\frac{1}{2} k z+\frac{1}{2} k z^{2}+\right. \\
& \left.\frac{1}{12} k^{2} z-\frac{1}{4} k^{2} z^{2}+\frac{1}{6} k^{2} z^{3}+. .\right) .
\end{aligned}
$$

Using $c=\frac{\sigma^{2}}{k}-\mu$ then the above equations become

$$
\begin{aligned}
\ln E^{*}\left[e^{z X(1)}\right]= & r z-\frac{1}{2} r k z+\frac{1}{2} r k z^{2}+\frac{1}{12} r k^{2} z \\
& -\frac{1}{4} r k^{2} z^{2}+\frac{1}{6} r k^{2} z^{3}-\frac{1}{2} z \sigma^{2}+\frac{1}{2} k z \mu \\
& +\frac{1}{2} z^{2} \sigma^{2}-\frac{1}{2} k z^{2} \mu+\frac{1}{12} k z \sigma^{2} \\
& -\frac{1}{12} k^{2} z \mu-\frac{1}{4} k z^{2} \sigma^{2}+\frac{1}{4} k^{2} z^{2} \mu \\
& +\frac{1}{6} k z^{3} \sigma^{2}-\frac{1}{6} k^{2} z^{3} \mu+. . \\
= & \mu^{*} z+\frac{1}{2} \sigma^{2} z^{2}+k P(z)
\end{aligned}
$$

with $\mu^{*}=r-\frac{1}{2} \sigma^{2} \quad$ and $\quad P(z)=\frac{1}{2}(r-\mu)\left(z^{2}-z\right)+$ $\sigma^{2}\left(\frac{1}{12} z-\frac{1}{4} z^{2}+\frac{1}{6} z^{3}\right)$, so the above equation can be converted into

$$
E\left[e^{z X(1)}\right]=\exp \left[\mu^{*} z+\frac{1}{2} \sigma^{2} z^{2}\right] \exp [k P(z)] \ldots
$$

We can notify that $\exp [k P(z)]=1+k P(z)+$ $\frac{1}{2} k^{2}(P(z))^{2}+\cdots$, so we have

$$
E^{*}\left[e^{z X(1)}\right]=\exp \left[\mu^{*} z+\frac{1}{2} \sigma^{2} z^{2}\right](1+k P(z)+\cdot \cdot
$$

In equation (13) it can be seen that $\exp \left[\mu^{*} z+\frac{1}{2} \sigma^{2} z^{2}\right]$ is a form of the moment generating function of standard normal random variable, $\exp \left[\mu^{*} Z+\frac{1}{2} \sigma^{2} z^{2}\right]=$ $E[\exp (z X)]$, where $\quad X=\sigma Z+\mu \quad$ with mean $\mu \quad$ and variance $\sigma^{2}$. So we get

$$
z \exp \left[\mu^{*} z+\frac{1}{2} \sigma^{2} z^{2}\right]=\frac{1}{\sigma} \int_{-\infty}^{\infty} z e^{z x} \phi\left(\frac{x-\mu^{*}}{\sigma}\right) d x .
$$

By using Maclaurin expansion and partial integral to the above equation, we obtained European call option pricing formula with Linear Approximation method, which can be written as

$$
\begin{aligned}
& S(0)\left[1-\Phi\left(\frac{2 \kappa-2 r-\sigma^{2}}{2 \sigma}\right)\right]-e^{-r} K[1- \\
& \left.\Phi\left(\frac{2 \kappa-2 r+\sigma^{2}}{2 \sigma}\right)\right]+ \\
& k e^{\kappa-r} S(0) \phi\left(\frac{2 \kappa-2 r+\sigma^{2}}{2 \sigma}\right)\left[\left(\frac{6 r-6 \mu-\sigma^{2}}{12 \sigma^{2}}\right)\left(\frac{2 \kappa-2 r+\sigma^{2}}{2 \sigma}\right)+\right. \\
& \left.\frac{1}{6 \sigma}\left[\left(\frac{2 \kappa-2 r+\sigma^{2}}{2 \sigma}\right)^{2}-1\right]\right]+ \\
& k e^{-r} K \phi\left(\frac{2 \kappa-2 r+\sigma^{2}}{2 \sigma}\right)\left[\left(\frac{6 r-6 \mu-\sigma^{2}}{12 \sigma}\right)-\right. \\
& \left(\frac{2 r-2 \mu-\sigma^{2}}{4 \sigma^{2}}\right)\left(\frac{2 \kappa-2 r+\sigma^{2}}{2 \sigma}\right)-\frac{1}{6 \sigma}\left[\left(\frac{2 \kappa-2 r+\sigma^{2}}{2 \sigma}\right)^{2}-\right. \\
& 1]]
\end{aligned}
$$

with :

$S(0)=$ Non-dividend stock price at time 0 $K=$ Exercise price 


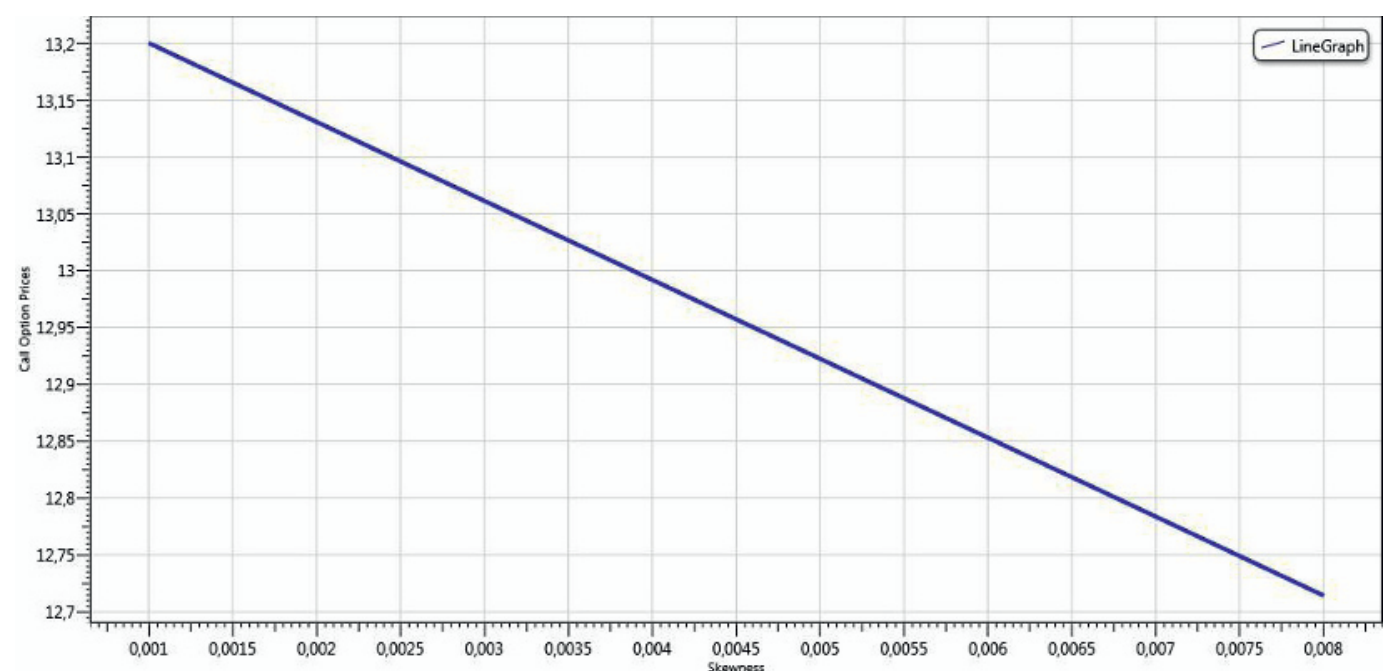

Figure 2. Option Price Calculation Using Linear Approximation Method.

$\kappa=\ln [K / S(0)]$

$r=$ Rate of interest

$\sigma^{2}=$ Variance

$\mu=$ Mean

$\Phi()=$. Cumulative standard normal distribution function

$k=\frac{\gamma}{\sigma^{2}}$

$\gamma=$ Skewness

$\phi()=$. Probability standard normal function

In the European call option pricing formula with Linear Approximation method (14) it can be seen that the first term on the formula that is,

$$
S(0)\left[1-\Phi\left(\frac{2 \kappa-2 r-\sigma^{2}}{2 \sigma}\right)\right]-e^{-r} K\left[1-\Phi\left(\frac{2 \kappa-2 r-\sigma^{2}}{2 \sigma}\right)\right]
$$

is a formula of the Black-Scholes model. Then the second term of the European call option pricing formula with Linear Approximation method is the rate of change between European call option pricing formula using Black-Scholes model, where $\gamma=0$, with a European call option pricing formula with Linear Approximation method, where $\gamma \neq 0$. This results in accordance with [11].

Table 1. Option Prices Comparison

\begin{tabular}{|c|c|c|c|}
\hline $\begin{array}{c}\text { Exercise } \\
\text { Price } \\
\text { (K) }\end{array}$ & $\begin{array}{c}\text { Black- } \\
\text { Scholes }\end{array}$ & $\begin{array}{c}\text { Shifted } \\
\text { Poisson }\end{array}$ & $\begin{array}{c}\text { Linear } \\
\text { Approximation }\end{array}$ \\
\hline 80 & 27.993 & 27.613 & 27.500 \\
\hline 85 & 23.864 & 23.089 & 23.220 \\
\hline 90 & 19.989 & 18.565 & 19.265 \\
\hline 95 & 16.439 & 15.696 & 15.742 \\
\hline 100 & 13.270 & 13.005 & 12.714 \\
\hline 105 & 10.515 & 10.315 & 10.196 \\
\hline 110 & 8.183 & 7.624 & 8.152 \\
\hline 115 & 6.258 & 6.418 & 6.522 \\
\hline 120 & 4.708 & 5.383 & 5.230 \\
\hline
\end{tabular}

\section{Results and Conclusion}

European call option price using Linear Approximation method for various values of skewness can be seen in Figure 2.

By comparing the Black-Scholes formula, Shifted Poisson model and the Linear Approximation with various exercise prices we obtained the option prices Table 1. Black-Scholes formula has generally been used by both academics and investors in the European call option price calculation. Limitations of this formula are the factors that affect the price of this option is assumed to have normal distribution, but in fact the underlying assets do not have normal distribution, in other words, the underlying asset is influenced by skewness factor $(\gamma)$. By using shifted Poisson models were modified BlackScholes formula, but for the value of skewness is close to zero oscillation occurs which complicates the calculation process. Improvisation of this model is convert it to a Linear form so for skewness is close to zero option price calculation still produce an accurate value, which is close to the Blcak-Scholes option price.

\section{References}

1. Martinkutè-Kaulienè, R. Business, Management and Education. 10 (2): 289-301. (2012)

2. Miller, L., \& Bertus, M., Business Education \& Accreditation. 5 (2013)

3. T.Sunaryo, Manajemen Resiko Finansial, (Salemba Empat, Jakarta, 2009)

4. Zhu, Z., \& Hanson, F. B, Social Science Research Network. (2013)

5. Gerber, H., \& Landry, B., North American Actuarial Journal. 1 (1997)

6. Black, F., \& Scholes, M., Journal of Political Economy. 81 (1973)

7. Hull, J. C., Options, Futures, and Other Derivatives. (Pearson Prentice Hall, New Jersey, 2012)

8. Gerber, H., \& Shiu, E. Insurance: Mathematics and Economics. 18 (1996) 
9. Boyle et al. Financial Economics: With Applicationsin Insurance and Pensions. (Schaumburg: in press, 1997)

10. Lin, S. Introductory Stochastic Analysis for Finance and Insurance. (John Willey \& Sons, Inc, Canada, 2009)

11. Gerber, H., \& Shiu, E., North American Actuarial Journal. 1 (1997) 